\title{
SIMPLE RIGHT CONJUGACY CLOSED LOOPS
}

\author{
MARK GREER
}

\begin{abstract}
We give a general construction for right conjugacy closed loops, using $G L(2, q)$ for $q$ a prime power. Under certain conditions, the loops constructed are simple, giving the first general construction for finite, simple right conjugacy closed loops. We give a complete description of the isomorphism classes for the construction, yielding an exact count of non isomorphic loops for each $q$.
\end{abstract}

\section{INTRODUCTION}

A loop $(Q, \cdot)$ consists of a set $Q$ with a binary operation $\cdot: Q \times Q \rightarrow Q$ such that (i) for all $a, b \in Q$, the equations $a x=b$ and $y a=b$ have unique solutions $x, y \in Q$, and (ii) there exists $1 \in Q$ such that $1 x=x 1=x$ for all $x \in Q$. We denote these unique solutions by $x=a \backslash b$ and $y=b / a$, respectively. Standard references in loop theory are [2, 16].

We say a subset $S$ of a group $G$ is closed under conjugation if $x^{-1} y x \in S$ for all $x, y \in S$. A loop $Q$ is a right conjugacy closed loop (or RCC loop) if $R_{Q}$ is closed under conjugation. That is, $R_{x}^{-1} R_{y} R_{x} \in R_{Q}$ for all $x, y \in Q$. Similarly, a loop $Q$ is left conjugacy closed (LCC) if $L_{x}^{-1} L_{y} L_{x} \in$ $L_{Q}$ for all $x, y \in Q$. Most of the literature on the one-sided conjugacy closed loops deals with left conjugacy closed loops [1, 3, 4, 13]. RCC loops are the more natural choice here since our permutations act on the right.

For (two-sided) CC-loops, the existence of nonassociative simple loops is settled in the negative by Basarab's Theorem [1]: The factor of a CC-loop by its (necessarily normal) nucleus is an abelian group. It follows that a simple CC-loop must have nucleus coinciding with the whole loop, hence is a group.

In the one-sided case, nonassociative simple RCC loops are known to exist. The first example occurring in the literature seems to be the simple Bol loop of exponent 2 and order 96 constructed by G. Nagy [11, because a right Bol loop of exponent 2 is necessarily an RCC loop. Other examples arose in the computer search for nonassociative, finite simple automorphic loops [9], since every RCC loops is a right automorphic loop.

Here we give the first general construction of a large class of nonassociative, finite simple RCC loops. Our construction by no means accounts for all such loops; for example, Nagy's Bol loop of exponent 2 does not fit this construction. Thus a full classification of finite simple RCC loops is still elusive. Nevertheless, using MACE4 [12] and the loops package for GAP [6, 14], we have found by exhaustive computer search that our construction accounts for all finite simple RCC loops up to order 15.

It turns out that our construction is isomorphic to a construction due to Hall for projective planes, called Hall Planes [8]. For each irreducible monic quadratic over a finite field, there is a unique Hall quasifield created. Hall planes of the same order turn out to be isomorphic [15. The multiplication loops in Hall's quasifields are isomorphic to the RCC loops constructed in this paper. However, we will have non-trivial isomorphism classes (Theorem 4.2). It is interesting to note another paper which relates quasifields to (one-sided) conjugacy closed loops is [10].

2010 Mathematics Subject Classification. 20N05.

Key words and phrases. conjugacy closed loops, simple loops, finite fields. 
In section $\S 2$, we review basic notions from loop theory. We also give some basic results concerning with RCC loops. In section $\S 3$, we give our construction for our loops (Theorem 3.2) and prove these loops are indeed RCC (Theorem 3.7). After proving several structural results about these RCC loops, we show our construction gives simple RCC loops (Theorem 3.10).

As previously stated, our construction may give isomorphic RCC loops for certain orders. We explain this phenomenon in $\S 4$, showing that isomorphisms reduce to automorphisms of finite fields (Theorem 4.1) and count the isomorphism classes (Theorem 4.2). Finally, we end with some open questions.

\section{Right Conjugacy Closed loops}

To avoid excessive parentheses, we use the following convention:

- multiplication · will be less binding than divisions $\backslash, /$.

- divisions are less binding than juxtaposition

For example $x y / z \cdot y \backslash x y$ reads as $((x y) / z)(y \backslash(x y))$.

In a loop $Q$, the left and right translations by $x \in Q$ are defined by $y L_{x}=x y$ and $y R_{x}=y x$ respectively. We thus have $\backslash, /$ as $x \backslash y=y L_{x}^{-1}$ and $y / x=y R_{x}^{-1}$. We define the right section of $Q$, $R_{Q}=\left\{R_{x} \mid x \in Q\right\}$, right multiplication group of $Q, \operatorname{Mlt}_{\rho}(Q)=\left\langle R_{x} \mid x \in Q\right\rangle$ and multiplication group of $Q, \operatorname{Mlt}(Q)=\left\langle R_{x}, L_{x} \mid x \in Q\right\rangle$. We define the inner mapping group of $Q, \operatorname{Inn}(Q)=$ $\operatorname{Mlt}(Q)_{1}=\{\theta \in \operatorname{Mlt}(Q) \mid 1 \theta=1\}$.

A bijection $\theta: Q \rightarrow Q$ is an automorphism if $(x y) \theta=x \theta \cdot y \theta$ for all $x, y \in Q$. Under composition, we define the automorphism group of $Q$ Aut $(Q)$. A triple $(\alpha, \beta, \gamma)$ of bijections of a loop $Q$ is an autotopism if for all $x, y \in Q, x \alpha \cdot y \beta=(x y) \gamma$. The set $\operatorname{Atp}(Q)$ of all autotopisms of $Q$ is a group under composition.

A subloop $N$ of $Q$ is normal $(N \unlhd Q)$ if for every $x, y \in Q$ we have $x N=N x, x(y N)=(x y) N$, $(N x) y=N(x y)$, and $x(N y)=(x N) y$. A loop $Q$ is simple if the only normal subloops are the trivial, $\{1\}$ and $Q$.

The following proposition, which will be useful later as it uses right translations to check whether a magma $(Q, \cdot)$ is indeed a loop.

Proposition 2.1. ([9]) Let $(Q, \cdot)$ be a magma with $1 \in Q$ an identity element. Then $Q$ is a loop if and only if $R_{x} R_{y}^{-1}$ is fixed point free for every $x, y \in Q$ with $x \neq y$ and $x, y \neq 1$.

Proof. This follows from Lemmas 2.1 and 2.2.

For a loop $Q$, we have the following subsets of interest:

$$
\begin{array}{ll}
\text { the left nucleus of } Q, & N_{\lambda}(Q)=\{a \in Q \mid a \cdot x y=a x \cdot y \forall x, y \in Q\}, \\
\text { the middle nucleus of } Q, & N_{\mu}(Q)=\{a \in Q \mid x \cdot a y=x a \cdot y \forall x, y \in Q\}, \\
\text { the right nucleus of } Q, & N_{\rho}(Q)=\{a \in Q \mid x \cdot y a=x y \cdot a \forall x, y \in Q\}, \\
\text { the nucleus of } Q, & N(Q)=N_{\lambda}(Q) \cap N_{\mu}(Q) \cap N_{\rho}(Q), \\
\text { the commutant of } Q, & C(Q)=\{a \in Q \mid x a=a x \forall x \in Q\}, \\
\text { the center of } Q, & Z(Q)=N(Q) \cap C(Q) .
\end{array}
$$

For a loop $Q$, the nuclei $N(Q), N_{\lambda}(Q), N_{\mu}(Q)$, and $N_{\rho}(Q)$ are all subloops of $Q$ and the center $Z(Q)$ is a normal subloop of $Q$. However, the commutant, $C(Q)$ need not be a subloop in general of $Q$.

Proposition 2.2. Let $Q$ be a loop. Then $a \in C(Q) \cap N_{\lambda}(Q) \Leftrightarrow R_{a} \in Z\left(M_{l}\left(t_{\rho}(Q)\right)\right.$.

Proof. Let $a \in C(Q) \cap N_{\lambda}(Q)$. Then $\forall x, y \in Q$,

$$
y R_{a} R_{x}=y a \cdot x=a y \cdot x=a \cdot y x=y x \cdot a=y R_{x} R_{a} .
$$


Hence, $R_{a} \in Z\left(\operatorname{Mlt}_{\rho}(Q)\right)$. Conversely, let $R_{a} \in Z\left(\operatorname{Mlt}_{\rho}(Q)\right)$. Then $a x=1 R_{a} R_{x}=1 R_{x} R_{a}=x a$. Hence $a \in C(Q)$. Moreover,

$$
a \cdot y x=y x \cdot a=y R_{x} R_{a}=y R_{a} R_{x}=y a \cdot x=a y \cdot x .
$$

Thus, $a \in C(Q) \cap N_{\lambda}(Q)$.

Proposition 2.3. For a loop $Q$, the following are equivalent:

(1) $Q$ is an $R C C$ loop,

(2) The following holds for all $x, y, z \in Q$ :

$$
R_{x}^{-1} R_{y} R_{x}=R_{x \backslash y x}
$$

(3) The following holds for all $x, y, z \in Q$ :

$$
(x y) z=(x z) \cdot z \backslash(y z) .
$$

(4) For all $x \in Q,\left(R_{a}, R_{a} L_{a}^{-1}, R_{a}\right) \in \operatorname{Atp}(Q)$.

Proof. If $Q$ is an RCC loop, then $\forall x, y \in Q$, we have $R_{x}^{-1} R_{y} R_{x}=R_{z} \Leftrightarrow R_{y} R_{x}=R_{x} R_{z}$. Hence, applying this to 1 gives $y x=x z$, and thus, $z=x \backslash y x$. Similarly, $\left(\mathrm{RCC}_{1}\right)$ holds if and only if $R_{y} R_{z}=R_{z} R_{z \backslash y z}$ for all $y, z \in Q$, which is clearly equivalent to $\left(\mathrm{RCC}_{2}\right)$. Finally, $\left(R_{a}, R_{a} L_{a}^{-1}, R_{a}\right) \in$ $\operatorname{Atp}(Q)$ is simply $\left(\mathrm{RCC}_{2}\right)$.

Proposition 2.4. Let $Q$ be a $R C C$ loop. Then

(i) $N_{\mu}(Q)=N_{\rho}(Q) \unlhd Q$ and

(ii) $C(Q) \leq N_{\lambda}(Q)$.

Proof. For $(i)$, note that

$$
\left(i d_{Q}, R_{a}, R_{a}\right)\left(R_{a}, L_{a}^{-1}, i d_{Q}\right)=\left(R_{a}, R_{a} L_{a}^{-1}, R_{a}\right) \in \operatorname{Atp}(Q) .
$$

Therefore, if $\left(i d_{Q}, R_{a}, R_{a}\right)$ or $\left(R_{a}, L_{a}^{-1}, i d_{Q}\right)$ is in $\operatorname{Atp}(Q)$, the other one is as well. For normality, see [3].

For $(i i)$, let $a \in C(Q)$. Then, using $\left(\overline{\mathrm{RCC}_{2}}\right)$, we have

$$
a x \cdot y=x a \cdot y=x y \cdot y \backslash(a y)=x y \cdot a=a \cdot x y \text {. }
$$

\section{Constructing Simple RCC loops}

Let $\mathbb{F}_{q}$ be the finite field of order where $q=p^{n}$ for a prime $p$ and some $n>0$. For a matrix $M$, let $\operatorname{Det}(M), \operatorname{Tr}(A)$, and $C h a r(M)$ denote the standard determinant, trace and characteristic polynomial of the matrix $M$. In this paper, all matrices will be of size $2 \times 2$ (i.e. $M \in G L(2, q)$ ), hence $\operatorname{Char}(M)=x^{2}-\operatorname{Tr}(M) x+\operatorname{Det}(M) \in \mathbb{F}_{q}[x]$.

Let $f(x)=x^{2}-r x+s$ be irreducible in $\mathbb{F}_{q}[x]$. For each $b \in \mathbb{F}_{q}$, define

$$
M_{(0, b)}=\left(\begin{array}{cc}
b & 0 \\
0 & b
\end{array}\right)
$$

and for $a \neq 0$,

$$
M_{(a, b)}=\left(\begin{array}{cc}
r-b & \frac{f(b)}{-a} \\
a & b
\end{array}\right) .
$$

Note that $\operatorname{Det}\left(M_{(a, b)}\right)=s$ and $\operatorname{Tr}\left(M_{(a, b)}\right)=r$ and thus $\operatorname{Char}\left(M_{(a, b)}\right)=f(x)$.

Lemma 3.1. Let $f(x)=x^{2}-r x+s$ be irreducible in $\mathbb{F}_{q}[x]$. The conjugacy class of all matrices in $G L(2, q)$ with characteristic polynomial $f(x)$ is precisely the set $\left\{M_{(a, b)} \mid a, b \in \mathbb{F}_{q}\right\}$ for $a \neq 0$. 
Proof. Note that if two elements of $G L(2, q)$ are conjugate then they both have the same characteristic polynomial, and hence for a $2 \times 2$ matrix, have the same determinant and trace [17. Now suppose $M=\left(\begin{array}{ll}c & d \\ a & b\end{array}\right)$ has $\operatorname{Char}(M)=f(x)$. Note that $a \neq 0$ since $f(x)$ is irreducible; otherwise, $M$ would have $c$ and $b$ as eigenvalues. Now $r=\operatorname{Tr}(M)=c+b$, so that $c=r-b$. Also, $s=\operatorname{Det}(M)=(r-b) b-d a$, and so $-d a=b^{2}-r b+s=f(b)$. Hence $d=f(b) /(-a)$. Therefore $M=M_{(a, b)}$ as claimed.

Let $f(x)=x^{2}-r x+s$ be irreducible in $\mathbb{F}_{q}[x]$. Let $Q=\mathbb{F}_{q}^{2} \backslash\{[0,0]\}$, written as a set of row vectors. Define a binary operation $\circ_{f}$ on $Q$ by

$$
[a, b] \circ_{f}[c, d]=[a, b] M_{(c, d)} .
$$

Note that

$$
\begin{array}{ll}
{[a, b] \circ_{f}[c, d]=\left[a(r-d)+b c, \frac{-a f(d)}{c}+b d\right]} & c \neq 0, \\
{[a, b] \circ_{f}[0, d]=[a d, b d]} & c=0 .
\end{array}
$$

It is clear that $\circ_{f}$ is closed on $Q$. Indeed, if $[a, b] \circ_{f}[c, d]=[0,0]$ and $c=0$, then either both $a=b=0$ or $d=0$.

For $c \neq 0$, if $d=0$, then $a r+b c=\frac{-a s}{c}=0$. Thus, either $a=0$ implying $b=0$ or $s=0$. For $d \neq 0$, we have

$$
r-d=\frac{-b c}{a}=-d+r-\frac{s}{d}
$$

implying $s=0$. Therefore, $[a, b] \circ_{f}[c, d]=[0,0]$ if and only if either $[a, b]=[0,0]$ or $[c, d]=[0,0]$.

Remark. To keep notation clear,

(1) $[x, y]$ denotes an element in $Q$;

(2) $R_{[x, y]}$ denotes the right translation by $[x, y]$;

(3) $M_{(x, y)}$ denotes the matrix associated with the right translation by $[x, y]$.

(4) maps on $Q$ act of the right (i.e. $x \theta$ ) and maps on $\mathbb{F}_{q}$ act on the left (i.e. $\theta(x)$ ).

Theorem 3.2. (Hall [7]) $\left(Q, \circ_{f}\right)$ is a loop with identity element $[0,1]$ with 0 and 1 being the additive and multiplicative identity in $\mathbb{F}_{q}$ respectively.

An isomorphic construction can be found in [7]. To keep this paper self-contained, we give our own proof of the following.

Proof. First note that $R_{\left(Q, \circ_{f}\right)}=\left\{M_{(a, b)} \mid a, b \in F_{q}\right\} \backslash\left\{M_{(0,0)}\right\}$ by the definition of $\circ_{f}$. That is, $R_{[a, b]}$ corresponds uniquely to $M_{(a, b)}$ by construction. Now, by Proposition 2.1, it is enough to show that each $R_{[y, z]} R_{[u, v]}^{-1}=M_{(y, z)} M_{(u, v)}^{-1}$ is fixed-point free.

Let $M_{(y, z)}, M_{(u, v)} \in R_{\left(Q, \circ_{f}\right)}$ and suppose $M_{(y, z)} M_{(u, v)}^{-1}$ has a fixed point. Then, $M_{(y, z)} M_{(u, v)}^{-1}$ has an eigenvalue of 1 . Let $g(x)=\operatorname{Char}\left(M_{(y, z)} M_{(u, v)}^{-1}\right)$. Then

$$
\begin{aligned}
g(x) & =x^{2}-\operatorname{Tr}\left(M_{(y, z)} M_{(u, v)}^{-1}\right) x+\operatorname{Det}\left(M_{(y, z)} M_{(u, v)}^{-1}\right), \\
0=g(1) & =1^{2}-\operatorname{Tr}\left(M_{(y, z)} M_{(u, v)}^{-1}\right)+\operatorname{Det}\left(M_{(y, z)} M_{(u, v)}^{-1}\right) \\
& =1-\operatorname{Tr}\left(M_{(y, z)} M_{(u, v)}^{-1}\right)+1 .
\end{aligned}
$$

Thus, $\operatorname{Tr}\left(M_{(y, z)} M_{(u, v)}^{-1}\right)=2$. Therefore, $g(x)=x^{2}-2 x+1=(x-1)^{2}$. Then, either $M_{(y, z)} M_{(u, v)}^{-1}=$ $\left(\begin{array}{ll}1 & 0 \\ 0 & 1\end{array}\right)$ or $M_{(y, z)} M_{(u, v)}^{-1}$ is similar to $\left(\begin{array}{ll}1 & 1 \\ 0 & 1\end{array}\right)$. In the first case, we have $M_{(y, z)}=M_{(u, v)}$. For the 
second, suppose $M_{(y, z)} \neq M_{(u, v)}$ and let $P \in G L(2, q)$ such that $P M_{(y, z)} M_{(u, v)}^{-1} P^{-1}=\left(\begin{array}{ll}1 & 1 \\ 0 & 1\end{array}\right)$. Then define $A=P M_{(y, z)} P^{-1}$ and

$B=P M_{(u, v)} P^{-1}$, so that $A B^{-1}=\left(\begin{array}{ll}1 & 1 \\ 0 & 1\end{array}\right)$. Note that $A$ and $B$ have the same determinant and trace as $M_{(y, z)}$ and $M_{(u, v)}$, respectively and hence $\operatorname{Char}(A)=\operatorname{Char}(B)=f(x)$. Let $A=$ $\left(\begin{array}{ll}a & b \\ c & d\end{array}\right), B=\left(\begin{array}{ll}e & f \\ g & h\end{array}\right)$. Then $[1,0] A=[1,1] B$ and $[0,1] A=[0,1] B$. Hence $a=e+g, b=f+h, c=$ $g, d=h$. Thus $A=\left(\begin{array}{cc}e+g & f+h \\ g & h\end{array}\right)$ and since $\operatorname{Tr}(A)=\operatorname{Tr}(B), g=0$. Hence, $A, B$ are upper triangular matrices and therefore $\operatorname{Char}(A)=f(x)$ is reducible, which is a contradiction.

Lemma 3.3. In $\left(Q, \circ_{f}\right)$

(i) for $a \neq 0, R_{[a, b]}^{-1}=M_{(a, b)}^{-1}=$

$$
\left(\begin{array}{cc}
r-b & \frac{f(b)}{-a} \\
a & b
\end{array}\right)^{-1}=\frac{1}{s}\left(\begin{array}{cc}
b & f(b) / a \\
-a & r-b
\end{array}\right)=\frac{1}{s} M_{[-a, r-b]},
$$

(ii) $R_{[0, b]}^{-1}=\frac{1}{b}\left(\begin{array}{cc}1 & 0 \\ 0 & 1\end{array}\right)$,

(iii) $R_{[a, b],[c, d]}=M_{(a, b)} M_{(c, d)} M_{[a, b] \circ_{f}[c, d]}^{-1}=$

$$
\left(\begin{array}{cc}
s & \frac{-\left(a^{2} s f(d)-a b c d s-a b c d+a b c r+a c d r-a c r^{2}+a c r s+c^{2} f(b)\right)}{(a c(b c-a d+a r))} \\
0 & 1
\end{array}\right),
$$

(iv) $R_{[a, b],[0, d]}=M_{(a, b)} M_{(0, d)} M_{[a, b] \circ_{f}[0, d]}^{-1}=\left(\begin{array}{cc}d^{2} & \frac{(d-1)(b-r+b d)}{a} \\ 0 & 1\end{array}\right)$,

(v) $R_{[0, b],[c, d]}=M_{(0, b)} M_{(c, d)} M_{[0, b] \circ_{f}[c, d]}^{-1}=\left(\begin{array}{cc}b^{2} & \frac{(b-1)(d-r+b d)}{c} \\ 0 & 1\end{array}\right)$ and

(vi) $R_{[0, b],[0, d]}=M_{(0, b)} M_{(0, d)} M_{[0, b] o_{f}[0, d]}^{-1}=\left(\begin{array}{ll}1 & 0 \\ 0 & 1\end{array}\right)$.

Proof. For $(i)$, simply note

$$
\begin{aligned}
& {[x, y]\left(\begin{array}{cc}
r-b & \frac{f(b)}{-a} \\
a & b
\end{array}\right)\left(\begin{array}{cc}
r-b & \frac{f(b)}{-a} \\
a & b
\end{array}\right)^{-1}} \\
& =\left[x(r-b)+a y, \frac{-x(f(b))}{c}+b y\right]\left(\begin{array}{cc}
\frac{b}{s} & \frac{f(b)}{s a} \\
\frac{-a}{s} & \frac{r-b}{s}
\end{array}\right) \\
& =[x, y] .
\end{aligned}
$$

Similarly, for $(i i)$. For $(i i i)$, using $(i)$, we have

$$
M_{(a, b) \circ_{f}(c, d)}^{-1}=\left(\begin{array}{cc}
\frac{\frac{-a f(d)}{c}+b d}{s} & \frac{f\left(\frac{-a f(d)}{c}+b d\right)}{s a} \\
\frac{-(a(r-d)+b c)}{s} & \frac{r-\left(\frac{-a f(d)}{c}+b d\right)}{s}
\end{array}\right) .
$$

Therefore, we have

$$
\left(\begin{array}{cc}
r-b & \frac{f(b)}{-a} \\
a & b
\end{array}\right)\left(\begin{array}{cc}
r-d & \frac{f(d)}{-c} \\
c & d
\end{array}\right)\left(\begin{array}{cc}
\frac{\frac{-a f(d)}{c}+b d}{s} & \frac{f\left(\frac{-a f(d)}{c}+b d\right)}{s a} \\
\frac{-(a(r-d)+b c)}{s} & \frac{r-\left(\frac{-a f(d)}{c}+b d\right)}{s}
\end{array}\right)
$$




$$
=\left(\begin{array}{cc}
s & \frac{-\left(a^{2} s f(d)-a b c d s-a b c d+a b c r+a c d r-a c r^{2}+a c r s+c^{2} f(b)\right)}{(a c(b c-a d+a r))} \\
0 & 1
\end{array}\right) .
$$

A similar calculation gives $(i v)$. Finally, $(v)$ and $(v i)$ follow from $(i v)$ and Lemma 2.2 ,

For a loop $Q, x$ has a two-sided inverse if $1 / x=x \backslash 1$, denoted $x^{-1}$. A loop is said to satisfy the right inverse property if $(y x) x^{-1}=y \Leftrightarrow R_{x^{-1}}=R_{x}^{-1}$ for all $x, y \in Q$. Note that a loop $Q$ satisfying RIP has two-sided inverses for all $x \in Q$.

Corollary 3.4. $\left(Q, \circ_{f}\right)$ satisfies RIP.

Proof. This follows quickly from (3.3) $(i)$ and $(i i)$.

Corollary 3.5. $|G L(2, q)|=\left|\left(Q, \circ_{f}\right)\right|\left|\operatorname{Inn}_{\rho}\left(Q, \circ_{f}\right)\right|$

Proof. Note that $\left|R_{\left(Q, o_{f}\right)}\right|=\left|\left(Q, \circ_{f}\right)\right|$. By Lemma 3.3. $\operatorname{Inn}_{\rho}\left(Q, \circ_{f}\right)=\left\{\left(\begin{array}{ll}x & y \\ 0 & 1\end{array}\right) \mid x, y \in \mathbb{F}_{q}\right\}$. Hence, for $A \in G F(2, q)$ where $A=\left(\begin{array}{ll}a & b \\ c & d\end{array}\right)$ for some $a, b, c, d \in \mathbb{F}_{q}, c \neq 0$, we have $A=B C$ where

$$
B=\left(\begin{array}{cc}
\frac{a s}{a d-b c} & \frac{a^{2} d^{2}-r a^{2} d+s a^{2}-2 a b c d+r a b c+b^{2} c^{2}}{b c^{2}-a c d} \\
\frac{c s}{a d-b c} & -\frac{a s-a d r+b c r}{a d-b c}
\end{array}\right) \quad C=\left(\begin{array}{cc}
\frac{a d-b c}{s} & \frac{a d^{2}+a s-b c d-a d r+b c r}{c s} \\
0 & 1
\end{array}\right)
$$

It is easy to see that $\operatorname{Det}(B)=s$ and $\operatorname{Tr}(B)=r$, and therefore, $B \in R_{\left(Q, \circ_{f}\right)}$. It is also clear that $C \in \operatorname{Inn}_{\rho}\left(Q, \circ_{f}\right)$. If $c=0$, then we have

$$
B=\left(\begin{array}{ll}
d & 0 \\
0 & d
\end{array}\right) \quad C=\left(\begin{array}{cc}
\frac{a}{d} & \frac{b}{d} \\
0 & 1
\end{array}\right)
$$

and it is easy to see that $A=\left(\begin{array}{ll}a & b \\ 0 & d\end{array}\right)=B C$

It is well known that the center of $G L(n, q)$ are scalar multiples of $I$ [17. Thus, we have the following:

Lemma 3.6. $C\left(Q, \circ_{f}\right)=\left\{[0, b] \mid \forall b \in \mathbb{F}_{q} b \neq 0\right\}$. That is, the only elements of $C\left(Q, \circ_{f}\right)$ are in the set $\left\{R_{[a, b]} \mid[a, b] \in C\left(Q, \circ_{f}\right)\right\}$. Moreover, $C\left(Q, \circ_{f}\right)$ is a subloop of $\left(Q, \circ_{f}\right)$.

Proof. Using Propositions 2.2, 2.4 and the above remark, we are done.

Now, the loop $\left(Q, \circ_{f}\right)$ has been constructed such that $R_{\left(Q, \circ_{f}\right)}$ is a union of conjugacy classes in $G L(2, q)$, namely the center $Z(G L(2, q)$ ) (scalar matrices) and the conjugacy class of matrices $M$ with $\operatorname{Char}(M)=f(x)$.

Theorem 3.7. $\left(Q, \circ_{f}\right)$ is an $R C C$ loop.

Proof. Let $[a, b] \in\left(Q, \circ_{f}\right)$. First, if $a=0$ then, $M_{(0, b)}=\left(\begin{array}{ll}b & 0 \\ 0 & b\end{array}\right)$ and

$$
[0, b] \in C(Q, \circ) \cap N_{\lambda}(Q, \circ) \Rightarrow R_{(0, b)} \in Z\left(M l t_{\rho}(Q, \circ)\right)
$$

by Proposition 2.2 and Lemma 3.6. Therefore, for any $[c, d] \in(Q, \circ)$,

$$
R_{[c, d]} R_{[0, b]} R_{[c, d]}^{-1}=M_{(c, d)} M_{(0, b)} M_{(c, d)}^{-1}=M_{(c, d)} M_{(c, d)}^{-1} M_{(0, b)}=M_{(0, b)}=R_{[0, b]} .
$$

Else, let $[c, d] \in(Q, \circ)$ and see that

$$
\begin{aligned}
& \operatorname{Det}\left(M_{(c, d)} M_{(a, b)} M_{(c, d)}^{-1}\right)=\operatorname{Det}\left(M_{(c, d)}\right) \operatorname{Det}\left(M_{(a, b)}\right) \operatorname{Det}\left(M_{(c, d)}^{-1}\right) \\
&=\operatorname{sss}^{-1}=s=\operatorname{Det}\left(M_{(a, b)}\right) . \\
& 6
\end{aligned}
$$


Similarly, $\operatorname{Tr}\left(M_{(c, d)} M_{(a, b)} M_{(c, d)}^{-1}\right)=r$. Hence $R_{[c, d]} R_{[a, b]} R_{[c, d]}^{-1} \in R_{(Q, \circ)}$.

Lemma 3.8. Let $q \neq 3$. Then $C\left(Q, \circ_{f}\right)=N_{\lambda}\left(Q, \circ_{f}\right)$. If $q=3$ and $r \neq 0$, then $C\left(Q, \circ_{f}\right)=$ $N_{\lambda}\left(Q, \circ_{f}\right)$. Finally, if $q=3$ and $r=0$, then $C\left(Q, \circ_{f}\right) \leq N_{\lambda}\left(Q, \circ_{f}\right)$.

Proof. For $q=2,\left|\left(Q, \circ_{f}\right)\right|=3$, and is an abelian group. Let $q>3$ and note that there exists a $d \in \mathbb{F}_{q}$ such that $d^{2} \neq 1$. Suppose $[x, y] \in N_{\lambda}\left(Q, \circ_{f}\right)$. Then for any $a \in \mathbb{F}_{q} \backslash\{0\}$,

$$
\left([x, y] \circ_{f}[a, 0]\right) \circ_{f}[0, d]=[x, y] \circ_{f}\left([a, 0] \circ_{f}[0, d]\right),
$$

or equivalently, $[x, y] R_{[a, 0],[0, d]}=[x, y]$. Hence, by Proposition $3.3(i v), d^{2} x=x$. But $d^{2} \neq 1$, and thus we have $x=0$. For $r \neq 0$, let $d \neq 1$. Then, as before, Proposition 3.3( $i v$ ) gives

$$
y-\frac{r x(d-1)}{a}=y .
$$

But $r \neq 0$ and hence, $x=0$. When $q=3$ and $r=0, C\left(Q, \circ_{f}\right)<N_{\lambda}(Q, \circ f)[\underline{6}$, 14].

Our goal is to construct simple RCC loops. Therefore, it is vital to understand the structure of normal subloops of an RCC loop. Let $Q$ be a RCC-loop with $N \unlhd Q$ and consider $R_{N}=\left\{R_{x} \mid\right.$ $x \in N\}$. Fix $x \in N$ and then $\forall y \in Q, R_{y} R_{x} R_{y}^{-1}=R_{(y x / y)} \in R_{N}$ since $y x / y \in N$. Hence, normal subloops of $Q$ correspond to unions of conjugacy classes in $R_{Q}$. That is, normal subloops of $Q$ correspond to unions of conjugacy classes of matrices in GL $(2, \mathrm{q})$ which are contained in $R_{\left(Q, \circ_{f}\right)}$. $R_{\left(Q, \circ_{f}\right)}$ itself is the union of conjugacy classes, namely, $\left\{M_{(a, b)} \mid a, b \in Q, a, b \neq 0\right\}$, which has size $q^{2}-q$, and the $q-1$ one-element conjugacy classes in the center of $G L(2, q)$. Since the order of a normal subloop of $Q$ must divide $|Q|=q^{2}-1$, we have the following.

Lemma 3.9. The only two non-trivial normal subgroups of $\left(Q, \circ_{f}\right)$ are $\{[0,1],[0,-1]\}$ and $C\left(Q, \circ_{f}\right)$.

Proof. Using the above remark, the only options are matrices of the form $\left(\begin{array}{ll}b & 0 \\ 0 & b\end{array}\right)$. Hence, either we have the $C\left(Q, \circ_{f}\right)$ or $\{[0,1],[0,-1]\} \leq C\left(Q, \circ_{f}\right)$.

Theorem 3.10. Let $f(x)=x^{2}-r x+s$ be irreducible. If $r \neq 0$, then $\left(Q, \circ_{f}\right)$ is simple. If $r=0$, then $Z\left(Q, \circ_{f}\right)=\{[0, \pm 1]\}$ and $\left(Q, \circ_{f}\right) / Z\left(Q, \circ_{f}\right)$ is simple.

Proof. Let $\operatorname{Tr}\left(M_{(a, b)}\right) \neq 0$ and suppose $\left(N, \circ_{f}\right) \unlhd\left(Q, \circ_{f}\right)$. Then, by Lemma 3.8, $\left(N, \circ_{f}\right) \leq$ $C\left(Q, \circ_{f}\right)=N_{\lambda}\left(Q, \circ_{f}\right)$. Fix $[0, z] \in\left(N, \circ_{f}\right)$ and let $[0, a],[0, c] \in\left(Q, \circ_{f}\right)$. Then

$$
[c, 0] \circ_{f}\left([a, 0] \circ_{f}[0, z]\right)=\left([c, 0] \circ_{f}[a, 0]\right) \circ_{f}[0, z] .
$$

Thus, $\left[c r, \frac{-c s}{a z}\right]=\left[c r z, \frac{-c s z}{a}\right]$. Hence $z=1$. That is, if

$$
\left(N, \circ_{f}\right) \unlhd\left(Q, \circ_{f}\right) \Leftrightarrow\left(N, \circ_{f}\right)=\{[0,1]\} .
$$

Therefore, the only normal subloops are trivial and $\left(Q, \circ_{f}\right)$ is simple.

Else, let $[a, b],[c, d] \in\left(Q, \circ_{f}\right)$ and $[0, z] \in\left(N, \circ_{f}\right)$ Note that

$$
M_{(a, b)}=\left(\begin{array}{cc}
-b & \frac{s+b^{2}}{-a} \\
a & b
\end{array}\right) \quad M_{(c, d)}=\left(\begin{array}{cc}
-d & \frac{s+d^{2}}{-c} \\
c & d
\end{array}\right) .
$$

Now,

implies

$$
[c, d] \circ_{f}\left([a, b] \circ_{f}[0, z]\right)=\left([c, d] \circ_{f}[a, b]\right) \circ_{f}[0, z] .
$$

$$
\left[z(a d-b c), b d z-\frac{c\left(b^{2} z^{2}+s\right)}{a z}\right]=\left[z(a d-b c), z\left(b d-\frac{c\left(b^{2}+s\right)}{a}\right)\right] .
$$

This is only solvable when $z= \pm 1$, and hence, $Z\left(Q, \circ_{f}\right)=\{[0, \pm 1]\}$. Therefore, $\left(Q, \circ_{f}\right)$ is not simple. However, $\left(Q, \circ_{f}\right) / Z\left(Q, \circ_{f}\right)$ is simple, since our same computation would for $z= \pm 1$ in 
$\left(Q, \circ_{f}\right) / Z\left(Q, \circ_{f}\right)$, but $[0,1]=[0,-1]$ in this loop. Thus, the only possible normal subloops are again trivial.

The following is an example for constructing a simple RCC loop of order 8 , from $G L(2,3)$.

Example 3.11. Let $q=3$, thus elements of $\left(Q, \circ_{f}\right)$ are

$$
\{1=[0,1], 2=[0,2], 3=[1,0], 4=[1,1], 5=[1,2], 6=[2,0], 7=[2,1], 8=[2,2]\} .
$$

Take $f(x)=x^{2}+2 x+2$, irreducible in $\mathbb{F}_{3}$. The conjugacy class of all matrices in $G L(2,3)$ with characteristic polynomial $f(x)$ are

$$
\left\{\left(\begin{array}{ll}
1 & 1 \\
1 & 0
\end{array}\right),\left(\begin{array}{ll}
0 & 1 \\
1 & 1
\end{array}\right),\left(\begin{array}{ll}
2 & 2 \\
1 & 2
\end{array}\right),\left(\begin{array}{ll}
1 & 2 \\
2 & 0
\end{array}\right),\left(\begin{array}{ll}
0 & 2 \\
2 & 1
\end{array}\right),\left(\begin{array}{ll}
2 & 1 \\
2 & 2
\end{array}\right)\right\}
$$

with the full set of matrices in $R_{\left(Q, \circ_{f}\right)}$

$$
\left\{\left(\begin{array}{ll}
1 & 0 \\
0 & 1
\end{array}\right),\left(\begin{array}{ll}
2 & 0 \\
0 & 2
\end{array}\right),\left(\begin{array}{ll}
1 & 1 \\
1 & 0
\end{array}\right),\left(\begin{array}{ll}
0 & 1 \\
1 & 1
\end{array}\right),\left(\begin{array}{ll}
2 & 2 \\
1 & 2
\end{array}\right),\left(\begin{array}{ll}
1 & 2 \\
2 & 0
\end{array}\right),\left(\begin{array}{ll}
0 & 2 \\
2 & 1
\end{array}\right),\left(\begin{array}{ll}
2 & 1 \\
2 & 2
\end{array}\right)\right\} .
$$

Note

$$
M_{(0,1)}=\left(\begin{array}{ll}
1 & 0 \\
0 & 1
\end{array}\right), M_{(0,2)}=\left(\begin{array}{ll}
2 & 0 \\
0 & 2
\end{array}\right) M_{(1,0)}=\left(\begin{array}{ll}
1 & 1 \\
1 & 0
\end{array}\right), \ldots
$$

Now, act on elements in $\left(Q, \circ_{f}\right)$ by the matrices above, giving the permutations for $R_{\left(Q, \circ_{f}\right)}$. For example, $M_{(2,2)}=\left(\begin{array}{ll}2 & 1 \\ 2 & 2\end{array}\right)$ gives the permutation $(1,8,6,5,2,4,3,7)$ since

$$
\begin{array}{lll}
{[0,1]\left(\begin{array}{ll}
2 & 1 \\
2 & 2
\end{array}\right)=[2,2],} & {[0,2]\left(\begin{array}{ll}
2 & 1 \\
2 & 2
\end{array}\right)=[1,1],} & {[1,0]\left(\begin{array}{ll}
2 & 1 \\
2 & 2
\end{array}\right)=[2,1],} \\
{[1,1]\left(\begin{array}{ll}
2 & 1 \\
2 & 2
\end{array}\right)=[2,1],} & {[1,2]\left(\begin{array}{ll}
2 & 1 \\
2 & 2
\end{array}\right)=[0,2],} & {[2,0]\left(\begin{array}{ll}
2 & 1 \\
2 & 2
\end{array}\right)=[1,2],} \\
{[2,1]\left(\begin{array}{ll}
2 & 1 \\
2 & 2
\end{array}\right)=[0,1],} & {[2,2]\left(\begin{array}{ll}
2 & 1 \\
2 & 2
\end{array}\right)=[2,0] .}
\end{array}
$$

Hence, we have

$$
\begin{aligned}
R_{\left(Q, \circ_{f}\right)}=\{( & ,(1,2)(3,6)(4,8)(5,7),(1,3,4,7,2,6,8,5),(1,4,5,6,2,8,7,3), \\
& (1,5,3,8,2,7,6,4),(1,6,7,4,2,3,5,8),(1,7,8,3,2,5,4,6), \\
& (1,8,6,5,2,4,3,7)\} .
\end{aligned}
$$

Since $r \neq 0,\left(Q, \circ_{f}\right)$ is simple and has the following multiplication table.

\begin{tabular}{c|cccccccc}
$\circ_{f}$ & 1 & 2 & 3 & 4 & 5 & 6 & 7 & 8 \\
\hline 1 & 1 & 2 & 3 & 4 & 5 & 6 & 7 & 8 \\
2 & 2 & 1 & 6 & 8 & 7 & 3 & 5 & 4 \\
3 & 3 & 6 & 4 & 1 & 8 & 5 & 2 & 7 \\
4 & 4 & 8 & 7 & 5 & 1 & 2 & 6 & 3 \\
5 & 5 & 7 & 1 & 6 & 3 & 8 & 4 & 2 \\
6 & 6 & 3 & 8 & 2 & 4 & 7 & 1 & 5 \\
7 & 7 & 5 & 2 & 3 & 6 & 4 & 8 & 1 \\
8 & 8 & 4 & 5 & 7 & 2 & 1 & 3 & 6
\end{tabular}

TABLE 1. Multiplication Table for $\left(Q, \circ_{f}\right)$ 


\section{ISOMORPhism Classes}

For $\mathbb{F}_{q}$, there are $\frac{q^{2}-q}{2}$ irreducible polynomials of degree 2 over $\mathbb{F}_{q}[5$. Hence, it is natural to assume we would create the same number of nonisomorphic RCC loops for a given $q$. This turns out not to be the case. For example, when $q=4$, there are 6 irreducible polynomials over $\mathbb{F}_{4}$ and we create 6 RCC loops associated to each polynomial. However, only 3 are nonisomorphic, and each simple. For $q=8$, we have only 10 nonisomorphic RCC loops, instead of 28 we can construct. The following table gives a count of RCC loops constructed from $G L(2, q)$. Note that RCC loops of order $p$ a prime are groups 3 . Our list is exhaustive for simple RCC loops up to and including order $15[6,12$, 14]. Also, for the loops of order 24, 10 loops are constructed from $G L(2,5)$ and 3 are constructed from $G L(2,7)$.

\begin{tabular}{|c|c|c|c|c|c|}
\hline$q$ & Order & $\begin{array}{l}\text { Number of } \\
\text { primitive } \\
\text { polynomials }\end{array}$ & $\begin{array}{c}\text { Number of } \\
\text { non-isomorphic, } \\
\text { nonassociative } \\
\text { RCC Loops }\end{array}$ & $\begin{array}{c}\text { Number of } \\
\text { Simple RCC loops }\end{array}$ & Exhaustive \\
\hline 3 & 8 & 3 & 3 & 2 & $\checkmark$ \\
\hline 5 & 12 & 2 & 2 & 2 & $\checkmark$ \\
\hline 4 & 15 & 6 & 3 & 3 & $\checkmark$ \\
\hline 5,7 & 24 & 10,3 & 13 & 11 & \\
\hline 9 & 40 & 2 & 2 & 2 & \\
\hline 7 & 48 & 21 & 21 & 18 & \\
\hline 11 & 60 & 5 & 5 & 5 & \\
\hline 8 & 63 & 28 & 10 & 10 & \\
\hline 9 & 80 & 36 & 18 & 16 & \\
\hline 13 & 84 & 6 & 6 & 6 & \\
\hline 11 & 120 & 55 & 55 & 50 & \\
\hline 13 & 168 & 78 & 78 & 72 & \\
\hline 16 & 255 & 120 & 30 & 30 & \\
\hline
\end{tabular}

TABLE 2. Table of RCC Loops

We now describe the isomorphism classes for this construction. It is well-known that $\alpha \in$ $\operatorname{Aut}\left(\mathbb{F}_{q}\right) \Leftrightarrow \alpha(x)=x^{p^{i}}$ for $0 \leq i \leq n$, the Frobenius automorphisms. Note that if $f(x)=x^{2}-r x+s$ is irreducible, then $g(x)=x^{2}-r^{p^{i}} x+s^{p^{i}}$ is irreducible as well.

Theorem 4.1. Let $f(x)=x^{2}-r_{1} x+s_{1}$ and $g(x)=x^{2}-r_{2} x+s_{2}$ be irreducible in $\mathbb{F}_{q}[x]$. Then $\phi:\left(Q, \circ_{f}\right) \rightarrow\left(Q, \circ_{g}\right)$ is an isomorphism if and only if $[a, b] \phi=[\alpha(a), \alpha(b)]$ for some $\alpha \in \operatorname{Aut}\left(\mathbb{F}_{q}\right)$.

Proof. Our first goal is to show $[0, b] \phi=[0, y]$ and $[a, 0] \phi=[x, 0]$ for some $a, b, x, y \in \mathbb{F}_{q}$.

Since $\phi$ is an isomorphism, $\phi$ maps $C\left(Q, \circ_{f}\right)$ to $C\left(Q, \circ_{g}\right)$, i.e. $[0, b] \phi=[0, y]$ for some $b, y \in \mathbb{F}_{q}$. Suppose $[a, 0] \phi=[x, y]$ and $[c, 0]=\left[x^{\prime}, y^{\prime}\right]$ for some $a, c, x, x^{\prime}, y, y^{\prime} \in \mathbb{F}_{q}$. Then

$$
\begin{gathered}
\left([a, 0] \circ_{f}[a, 0]\right) \phi=\left[r_{1}, s_{1}\right] \phi=\left[r_{2}, y r_{2}-s_{2}\right]=[x, y] \circ_{g}[x, y]=[a, 0] \phi \circ_{g}[a, 0] \phi \\
\left([c, 0] \circ_{f}[c, 0]\right) \phi=\left[r_{1}, s_{1}\right] \phi=\left[r_{2}, y^{\prime} r_{2}-s_{2}\right]=\left[x^{\prime}, y^{\prime}\right] \circ_{g}\left[x^{\prime}, y^{\prime}\right]=[c, 0] \phi \circ_{g}[c, 0] \phi
\end{gathered}
$$

Therefore, $y r_{2}-s_{2}=y^{\prime} r_{2}-s_{2}$ implying $y=y^{\prime}$. If $y \neq 0$, consider $b \neq 1$ and suppose $[a, 0] \phi=[x, y]$ for some $x \in \mathbb{F}_{q}$. We have

$$
\left([a, 0] \circ_{f}[0, b]\right) \phi=[a b, 0] \phi=[u, y]
$$

for some $u \in \mathbb{F}_{q}$. Similarly, supposing $[0, b] \phi=[0, v]$ for some $v \in \mathbb{F}_{q}$,

$$
[a, 0] \phi \circ_{g}[0, b]=[x, y] \circ[0, v]=[x v, y v] .
$$


But this implies $v=1$, so $[0, b] \phi=[0,1]$. Therefore, $b=1$ since $\phi$ is an isomorphism, a contradiction. Thus $[a, 0] \phi=[x, 0]$ for some $x \in \mathbb{F}_{q}$.

We show that $[a, b] \phi=[\alpha(a), \beta(b)]=[\alpha(a), \alpha(b)]$ for some bijections $\alpha, \beta$ of $\mathbb{F}_{q}$.

Suppose $[a, b] \phi=[x, y]$ and $[c, b] \phi=\left[z, y^{\prime}\right]$ for some $a, b, c, x, y, y^{\prime}, z \in \mathbb{F}_{q}$. Then

$$
\begin{aligned}
& \left([a, b] \circ_{f}[a, b]\right) \phi=\left[r_{1}, r_{1} b-s_{1}\right] \phi=\left[r_{2}, y r_{2}-s_{2}\right]=[x, y] \circ_{g}[x, y]=[a, b] \phi \circ_{g}[a, b] \phi \\
& \left([c, b] \circ_{f}[c, b]\right) \phi=\left[r_{1}, r_{1} b-s_{1}\right] \phi=\left[r_{2}, y^{\prime} r_{2}-s_{2}\right]=\left[z, y^{\prime}\right] \circ_{g}\left[z, y^{\prime}\right]=[c, b] \phi \circ_{g}[c, b] \phi
\end{aligned}
$$

Hence, $y=y^{\prime}$.

On the other hand, suppose $[a, b] \phi=[x, y],[a, c] \phi=\left[x^{\prime}, z\right]$, and $[1,0] \phi=[u, 0]$ for some $a, b, c, x, x^{\prime}, y, z, u \in \mathbb{F}_{q}$. Then

$$
\begin{aligned}
\left([a, b] \circ_{f}[1,0]\right) \phi & =\left[a r_{1}+b,-a s_{1}\right] \phi=[w, v] \\
& =\left[x r_{2}+y u, \frac{-x s_{2}}{u}\right]=[x, y] \circ_{g}[u, 0]=[a, b] \phi \circ_{g}[1,0] \phi, \\
\left([a, c] \circ_{f}[1,0]\right) \phi & =\left[a r_{1}+c,-a s_{1}\right] \phi=\left[w^{\prime}, v\right] \\
& =\left[x^{\prime} r_{2}+z u, \frac{-x^{\prime} s_{2}}{u}\right]=\left[x^{\prime}, z\right] \circ_{g}[u, 0]=[a, c] \phi \circ_{g}[1,0] \phi .
\end{aligned}
$$

Hence, $\frac{-x s_{2}}{u}=v=\frac{-x^{\prime} s_{2}}{u}$, thus $x=x^{\prime}$.

Thus we have shown $[a, b] \phi=[\alpha(a), \beta(b)]$. Since $\phi$ is an isomorphism, we have $\alpha, \beta$ bijections of $\mathbb{F}_{q}$ Moreover, $\alpha(0)=0$ and $\beta(1)=1$. Now,

$$
\begin{aligned}
{[\alpha(a b), \beta(0)] } & =[a b, 0] \phi \\
& =\left([a, 0] \circ_{f}[0, b]\right) \phi \\
& =[\alpha(a), \beta(0)] \circ_{g}[\alpha(0), \beta(b)] \\
& =[\alpha(a), \beta(0)] \circ_{g}[0, \beta(b)] \\
& =[\alpha(a) \beta(b), \beta(0) \beta(b)]
\end{aligned}
$$

Hence $\beta(0)=0$ and $\alpha(a) \beta(b)=\alpha(a b)$. But this is true for all $a, b \in \mathbb{F}_{q}$, thus setting $a=1$, we have $\alpha(b)=\beta(b)$ for all $b \in \mathbb{F}_{q}$. Thus, $[a, b] \phi=[\alpha(a), \alpha(b)]$ with $\alpha(a b)=\alpha(a) \alpha(b), \alpha(0)=0$ and $\alpha(1)=1$. Therefore, $\alpha$ is an automorphism of the multiplication group $\mathbb{F}_{q}^{*}$, and hence must be of the form $\alpha(x)=x^{k}$ for some $k$ since $\mathbb{F}_{q}^{*}$ is cyclic.

Thus,

$$
\begin{gathered}
\left([1,0] \circ_{f}[1,0]\right) \phi=\left[r_{1}, s_{1}\right] \phi=\left[r_{1}^{k}, s_{1}^{k}\right], \\
{[1,0] \phi \circ_{g}[1,0] \phi=[1,0] \circ_{g}[1,0]=\left[r_{2}, s_{2}\right]}
\end{gathered}
$$

Therefore $\alpha\left(r_{1}\right)=r_{2}$ and $\alpha\left(s_{1}\right)=s_{2}$. But $g(x)=x^{2}-r_{2} x+s_{2}=x^{2}-\alpha\left(r_{1}\right)(x)+\alpha\left(s_{1}\right)$ is irreducible, so $\alpha(x)=x^{p^{i}}$ a Frobenius map, that is, $\alpha \in \operatorname{Aut}\left(\mathbb{F}_{q}\right)$.

The reverse direction is obvious, since $\left([a, b] \circ_{f}[c, d]\right) \phi=[a, b] \phi \circ_{g}[c, d] \phi$ where $[a, b] \phi=[\alpha(a), \alpha(b)]$ with $\alpha \in \mathbb{F}_{q}$.

Note that $\left|\operatorname{Aut}\left(\mathbb{F}_{q}\right)\right|=n$ for $q=p^{n}$, so one would expect the number of nonisomorphic RCC loops constructed to be $\frac{q^{2}-q}{2 n}$. However, it is often the case that $\frac{q^{2}-q}{2 n} \notin \mathbb{N}$. Hence, we have the following.

Theorem 4.2. Let $p$ be a prime number and $q=p^{n}$. The number of nonisomorphic RCC loops constructed from $G L(2, q)$ is $\left\lfloor\frac{q^{2}-q}{2 n}\right\rfloor+\left(\frac{q^{2}-q}{2} \bmod n\right)$.

Proof. This follows quickly from Theorem 4.1 and the above note. 
Hence, we construct $\frac{p^{2}-p}{2}$ distinct RCC loops from $\mathbb{F}_{p}, \frac{p^{4}-p^{2}}{4}$ distinct RCC loops from $\mathbb{F}_{p^{2}}$, etc. For $\mathbb{F}_{8}, \frac{8^{2}-8}{6} \notin \mathbb{Z}$, so we have $\left\lfloor\frac{8^{2}-8}{6}\right\rfloor=9$ and $\left(\frac{8^{2}-8}{2} \bmod 3\right)=1$, and thus we have $9+1=10$ nonisomorphic RCC loops.

Lastly, we consider

Question 4.3. What group is $\operatorname{Mlt}_{\rho}\left(Q, \circ_{f}\right)$ ?

We have $M_{l}(Q)=\operatorname{Inn}_{\rho}(Q) \cdot R_{Q}$. Indeed, for $\theta \in \operatorname{Mlt}_{\rho}(Q)$ set $a=1 \theta$. Then $\psi=\theta R_{a}^{-1}$ fixes 1 , hence is an element of $\operatorname{Inn}_{\rho}(Q)$. Therefore, $\theta=\psi R_{a}$ and since $\operatorname{Inn}_{\rho}(Q) \cap R_{Q}=\iota$, we have the factorization.

Conjecture 4.4. $\operatorname{Inn}_{\rho}\left(Q, \circ_{f}\right)=\left\{\left(\begin{array}{ll}x & y \\ 0 & 1\end{array}\right) \mid x=a^{2} s^{m} \quad a, y \in \mathbb{F}_{q} \quad m \in \mathbb{Z}\right\}$.

We know $x$ must have this form from Lemma 3.3. The question is whether we can have any value for $y \in \mathbb{F}_{q}$. We do have the following.

Lemma 4.5. Let $H=\left\{\left(\begin{array}{ll}x & y \\ 0 & 1\end{array}\right) \mid x, y \in \mathbb{F}_{q}\right\}$. Then $G L(2, q)=R_{\left(Q, \circ_{f}\right)} \cdot H$.

Proof. Note that $\left|R_{\left(Q, \circ_{f}\right)}\right|=q^{2}-1$ and $|H|=q(q-1)$. We have $|G L(2, q)|=\left(q^{2}-1\right)\left(q^{2}-q\right)=$ $q(q+1)(q-1)^{2}=\left|R_{Q}\right||H|$. Since $R_{\left(Q, \circ_{f}\right)} \cap H=\left(\begin{array}{ll}1 & 0 \\ 0 & 1\end{array}\right)$, we have the desired result.

Hence, Question 4.3 reduces to what subgroups of $H$ can occur as $\operatorname{Inn}_{\rho}\left(Q, \circ_{f}\right)$ ?

Acknowledgment. Some investigations in this paper were assisted by the finite model builder MACE4 developed by McCune [12]. Similarly, all presented examples were verified using the GAP system [6] together with the LOOPS package [14].

\section{REFERENCES}

1. A.S. Basarab, Klass LK-lup, Matematicheskie issledovanija 120 (1991), 3-7.

2. R.H. Bruck, A Survey of Binary Systems, Springer-Verlag, Berlin, 1971.

3. A. Drápal, On multiplication groups of left conjugacy closed loops, Comment. Math. Univ. Carolinae 45 (2003), $223-236$.

4. - On left conjugacy closed loops with a nucleus of index two, Abhandlungen Aus Dem Mathematischen Seminar Der Universitat Hamburg 74 (2004), 205-221.

5. D.S. Dummit and R.M. Foote, Abstract algebra, John Wiley and Sons, Inc., Hoboken, NJ, 2004.

6. The GAP Group, Groups, Algorithms, and Programming, http://www.gap-system.org (2008).

7. M. Hall, Finite projective planes, The American Mathematical Monthly 62 (1955), no. 7, 18-24.

8. _ Theory of groups, vol. 1778, MacMillion Co., 1959.

9. K. W. Johnson, M.K. Kinyon, G.P. Nagy, and P. Vojtěchovský, Searching for small simple automorphic loops, LMS Journal of Computation and Mathematics 14 (2011), 200-213.

10. I. Matievics, Geometries over Universal Left Conjugacy Closed Quasifields, Geometriae Dedicata 65 (1997), $127-133$.

11. G.P. Nagy, A class of finite simple Bol loops of exponent 2, Trans. Amer. Math. Soc. 361 (2009), 5331-5343.

12. W.W. McCune, Prover9, Mace4, http://www.cs.unm.edu/〜mccune/prover9/ (2009).

13. G.P. Nagy and K. Strambach, Loops as invariant section in groups, and their geometry, Canad. J. Math. 46 (1994), 1027-1056.

14. G.P. Nagy and P. Vojtěchovský, Loops: Computing withquasigroups and loops, http://www.math.du.edu/loops (2008).

15. S.B. Nesbitt-Stobert and C.W.L. Garner, A direct proof that all hall planes of the same finite order are isomorphic, Riv. Mat. Univ. Parma 12 (1986), no. 4, 241-247.

16. H.O. Pflugfelder, Quasigroups and Loops: Introduction, Sigma Series in Pure Math, Berlin, 1990.

17. J.J. Rotman, An introduction to the theory of groups, 148, Springer-Verlag Graduate Texts in Mathematics, 1994. 
E-mail address: mgreer@una.edu

Department of Mathematics, One Harrison Plaza, University of North Alabama, Florence, Al 35632 USA 